\title{
Inner-Sphere Electron-Transfer Single lodide Mechanism for Dye Regeneration in Dye-Sensitized Solar Cells
}

\author{
Jiwon Jeon, ${ }^{\dagger}$ William A. Goddard, III, ${ }^{*},+, \$, \$$ and Hyungjun Kim ${ }^{*}, \dagger$ \\ ${ }^{\dagger}$ Graduate School of Energy Environment Water Sustainability (EEWS), Korea Advanced Institute of Science and Technology \\ (KAIST), Daejeon 305-701, Republic of Korea \\ ${ }^{\ddagger}$ Materials and Process Simulation Center, California Institute of Technology, Pasadena, California 91125, United States
}

Supporting Information

\begin{abstract}
During the regeneration of the oxidized dye in dye-sensitized solar cells, the redox couple of $\mathrm{I}^{-} / \mathrm{I}_{3}{ }^{-}$ reduces the photo-oxidized dye. The simplest mechanism would be a direct charge-transfer mechanism from $\mathrm{I}^{-}$to $\mathrm{D}^{+}$ $\left[\mathrm{D}^{+}+\mathrm{I}^{-} \rightarrow \mathrm{D}^{0}+\mathrm{I}\right]$, called the single iodide process (SIP). However, this is an unfavorable equilibrium because the redox potential of $\mathrm{I}^{\bullet} / \mathrm{I}^{-}$is $1.224 \mathrm{~V}$ vs SHE, which is $0.13 \mathrm{~V}$ higher than that of the dye. This led to the postulation of the two iodide process (TIP) $\left[\left(\mathrm{D}^{+} \cdots \mathrm{I}^{-}\right)+\mathrm{I}^{-} \rightarrow\left(\mathrm{D} \cdots \mathrm{I}_{2}^{-}\right)\right.$ $\left.\left.\rightarrow \mathrm{D}^{0}+\mathrm{I}_{2}^{-}\right)\right]$for a sufficiently high reducing power, but TIP is not consistent with either the recent experimental data suggesting the first-order kinetics or recent timeresolved spectroscopic measurements. To resolve this conundrum, we used quantum mechanics including Poisson-Boltzmann solvation to examine the electrontransfer process between $\mathrm{I}^{-}$and $\mathrm{D}^{+}$for the $\mathrm{Ru}(\mathrm{dcb})_{2} \mathrm{NCS}_{2}$ or N3 dye. We find that $\mathrm{I}^{-}$is attracted to the oxidized dye, positioning $\mathrm{I}^{-}$next to the NCS. At this equilibrium position, the $\mathrm{I}^{-}$electron is already $40 \%$ transferred to the NCS, showing that the redox potential of $\mathrm{I}^{-}$is well matched with the dye. This matching of the redox potential occurs because $\mathrm{I}^{-}$is partially desolvated as it positions itself for the inner-sphere electron transfer (ISET). The previous analyses all assumed an outersphere electron-transfer process. Thus our ISET-SIP model is consistent with the known redox potentials and with recent experimental reports. With the ISET-SIP mechanism, one can start to consider how to enhance the dye regeneration kinetics by redesigning ligands to maximize the interaction with iodide.
\end{abstract}

Cince Gratzel and O'Regan reported a successful dye$\checkmark$ sensitized solar cell (DSSC) in 1991, ${ }^{1}$ DSSCs have been considered as promising alternatives to photovoltaic devices due to their low cost of manufacturing and their relatively high energy conversion efficiency of $\sim 11 \%{ }^{2}$ The light-harvesting mechanism in the DSSC dye molecule resembles the photosynthetic process for converting solar energy to chemical energy, in which photoexcitation is followed rapidly by an efficient charge-carrier transport process.

The typical DSSC contains a ruthenium-based dye attached to a $\mathrm{TiO}_{2}$ anode which is immersed in an electrolyte containing an $\mathrm{I}^{-} / \mathrm{I}_{3}{ }^{-}$redox couple. After the dye absorbs a photon to excite the d-electron occupied in the $t_{2 g}$-like orbital of the dye to the lowest unoccupied molecular orbital (LUMO), forming $\mathrm{D}^{*}$, the excited electron is injected quickly $\left(<100 \mathrm{fs}^{3-6}\right)$ from the LUMO into the conduction band of the $\mathrm{TiO}_{2}$ while the hole remains localized on the dye, resulting in an oxidized dye, $\mathrm{D}^{+}$:

$$
\mathrm{D}^{0}+h \nu \rightarrow \mathrm{D}^{+}+\mathrm{e}^{-}\left(\mathrm{TiO}_{2}\right)
$$

The oxidized dye $\left(\mathrm{D}^{+}\right)$is then recovered by the redox couple $\left(\mathrm{I}^{-} / \mathrm{I}_{3}^{-}\right)$in the electrolyte, which transports the electron from the cathode to the dye. Transient ambient spectroscopy experiments show that dye regeneration takes $1-100 \mu \mathrm{s}$, $4,7,8$ which is much slower than the electron injection from the excited dye molecule to the $\mathrm{TiO}_{2}$ conduction band., 9

As a possible dye regeneration mechanism, the simplest one would be a one-step electron transfer from a single iodide to $\mathrm{D}^{+}$, namely the single iodide process (SIP). Although we should note the possible experimental uncertainty in the measurements of the kinetic order, many previous dye regeneration experiments found the rate to be linear in terms of the $\mathrm{I}^{-}$ concentration, ${ }^{7,11,12}$ which is consistent with SIP.

However, the possibility of SIP has often been ruled out ${ }^{9,13}$ based on the experimental standard reduction potential, $E^{0}\left(\mathrm{I}^{\bullet} /\right.$ $\left.\mathrm{I}^{-}\right)=1.224 \mathrm{~V}$ vs $\mathrm{SHE}^{14}$ which is $0.13 \mathrm{~V}$ higher than the $E^{0}$ value of the dye $\left(1.094 \mathrm{~V}\right.$ vs SHE). ${ }^{15}$ Rather, it has been suggested that at least two iodides must be incorporated to attain the dye regeneration process (two iodide process, TIP), ${ }^{16}$ where the bond formation between two iodides produces an electron with a high energy $\left(E^{0}\left(\mathrm{I}_{2}^{-\bullet} / \mathrm{I}^{-}\right)=0.784 \mathrm{~V}\right) .{ }^{13}$ However, this requires a three-body collision among $\mathrm{D}^{+}$and two iodides, which seems unlikely at the concentrations involved. Consequently, it has been suggested that a $\left(\mathrm{D}^{+} \ldots \mathrm{I}^{-}\right)$ complex is formed during the regeneration process, which is followed by a subsequent second iodide to reduce $\mathrm{D}^{+} .{ }^{16,17}$

However, we should note that the higher standard reduction potential simply implies that the system has an unfavorable equilibrium, but this does not necessarily mean that the reaction is thermodynamically prohibited. Also it is questionable if the equilibrium assertion would be valid under the operational condition of DSSC; thus, the possibility of SIP should not be excluded solely based on a thermodynamic argument. ${ }^{18-20}$ Indeed, recent time-resolved spectroscopic measurements suggest that the electron transfer from the

Received: November 30, 2012

Published: February 5, 2013 
iodide to the dye molecule precedes the I-I bond formation step, supporting our QM results and the SIP mechanism. ${ }^{20}$

In order to design new DSSC systems with improved efficiency, we need to determine the redox mechanism and kinetics of dye regeneration. Numerous experimental ${ }^{12,16,21,22}$ and theoretical ${ }^{23-26}$ studies have been carried out to understand the dye regeneration redox mechanism, but there remains considerable uncertainty regarding the details of the dye regeneration mechanism. ${ }^{9,18,19,25}$

To investigate the interaction between $\mathrm{D}^{+}$and $\mathrm{I}^{-}$, we carried out quantum mechanics (QM) calculations using the B3LYP flavor of density functional theory (DFT) combined with the Poisson-Boltzmann implicit solvation method $(\mathrm{PBF})^{27}$ incorporated in Jaguar. ${ }^{28}$ We used the B3LYP exchangecorrelation functional because our calculations (vide infra) show that this leads to accurate values for the reducing power of the iodide.

To describe the acetonitrile (AN) solvent, we used the standard dielectric constant of 37.5 and the standard solvent radius of $2.19 \AA^{29}$ For isolated ions such as iodide, the continuum solvation description depends critically on the ion radius. Thus we set the ionic radius of the iodine atom to 2.73 $\AA$, leading to $\Delta G^{\circ}{ }_{s}\left(\mathrm{I}^{-}\right)=-55.20 \mathrm{kcal} / \mathrm{mol}$ (cf. experimental value of $\left.-55.90 \mathrm{kcal} / \mathrm{mol}^{30}\right)$ and $\Delta G^{\circ}{ }_{s}\left(\mathrm{I}_{3}{ }^{-}\right)=-37.36 \mathrm{kcal} / \mathrm{mol}$ (cf. experimental value of $-38.40 \mathrm{kcal} / \mathrm{mol}{ }^{30}$ see Table S1).

For the initial locations of iodide with respect to the dye, we started with five distinct sites and optimized the structures including PBF solvation by the AN solution (Figure S1):

- site-SD: starts with $\mathrm{I}^{-}$located between the NCS ligand and the dicarboxylic-bipyridine $(\mathrm{dcb})$ ligand of $\mathrm{D}^{+}$

- site-DD: starts with $\mathrm{I}^{-}$located between two dcb ligands of $\mathrm{D}^{+}$

- site-COOH: starts with $\mathrm{I}^{-}$located near the carboxylic group of one dcb ligand of $\mathrm{D}^{+}$

- site-NCS: starts with $\mathrm{I}^{-}$located linearly along one of the NCS ligands of $\mathrm{D}^{+}$

- site-SS: starts with $\mathrm{I}^{-}$located between the two NCS ligands of $\mathrm{D}^{+}$

We found that the initial structures of site-NCS and site-SS both optimize to form site-SD, while site-DD and site- $\mathrm{COOH}$ remain nearly identical after minimization of the solution phase. The final geometries are shown in Figure 1.

The predicted binding energy of site-SD is $3.61 \mathrm{kcal} / \mathrm{mol}$ with respect to separately solvated $\mathrm{D}^{+}$and $\mathrm{I}^{-}$, much more favorable than the $0.86 \mathrm{kcal} / \mathrm{mol}$ binding for site-DD. We should note that our calculations treated the four carboxylic acids as neutral in order to avoid a large charge of the cluster

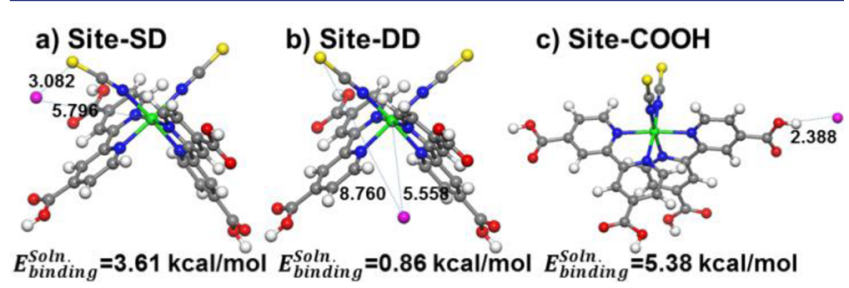

Figure 1. Final optimized geometry of $\mathrm{D}^{+}$and $\mathrm{I}^{-}$in acetonitrile (AN): (a) site-SD (started between one dcb and one NCS), (b) site-DD (started between the two dcb's), and (c) site- $\mathrm{COOH}$ (started at the $\mathrm{OH}$ of the $\mathrm{COOH}$ on one of the dcb's). The binding energy ( $\left.E_{\text {binding }}^{\text {soln }}\right)$ with respect to the separately solvated $\mathrm{D}^{+}$and $\mathrm{I}^{-}$is $3.61 \mathrm{kcal} / \mathrm{mol}$ for site-SD, $0.86 \mathrm{kcal} / \mathrm{mol}$ for site-DD, and $5.38 \mathrm{kcal} / \mathrm{mol}$ for site-COOH. that may lead to inaccuracy in the calculated solvation energies. However, in the experiments, most likely two of them bind to the $\mathrm{TiO}_{2}$ anode where iodine can hardly interact with. ${ }^{31}$ Supposing the other two carboxylic groups remain as protonated, these site- $\mathrm{COOH}$ with higher binding energy can compete with the site-SD allowing more fast electron transfer as will be discussed hereinafter. This suggests that the more deprotonated dye would be beneficial for the faster dye regeneration, which might ascribe the better efficiency of N719 dye rather than that of $\mathrm{N} 3$ dye. ${ }^{31-33}$

The singly occupied molecular orbitals (SOMOs) from the QM are shown in Figure 2. Figure 2a shows that the SOMO of
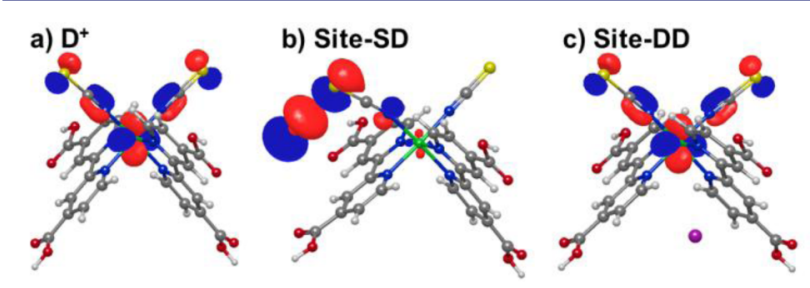

Figure 2. (a) Singly occupied molecular orbital (SOMO) of the oxidized dye solvated with AN. The hole left after photoexcitation and the transfer of the excited electron to the $\mathrm{TiO}_{2}$ localizes onto the $\mathrm{d}_{x y}{ }^{-}$ orbital of $\mathrm{Ru}$ and the two NCS ligands. (b) SOMO for the QMoptimized geometry (site-SD) for the $\left(\mathrm{D}^{+} \ldots \mathrm{I}^{-}\right)$complex solvated with AN. We see that the p-orbital of the iodide points toward the $S$ of the NCS, where the hole state is located, leading to a fast inner-sphere electron transfer (ISET). (c) SOMO for the QM-optimized geometry (site-DD) for the $\left(\mathrm{D}^{+} \cdots \mathrm{I}^{-}\right)$complex solvated with AN. Here there is no ISET.

the $\mathrm{D}^{+}$is delocalized over the two NCS ligands and the Ru $\mathrm{d}_{x y}$ orbital (where the $x y$ plane contains the two NCS ligands). The NCS ligands lead to a significant transfer of this hole characteristic onto the NCS and away from the $\mathrm{Ru}$, making it much more accessible to $\mathrm{I}^{-}$. As the hole is symmetric between the two NCS ligands, it can be transferred to either side.

At the optimum position of $\mathrm{I}^{-}$, Figure $2 \mathrm{~b}$ shows that the hole is equally on I and the nearby NCS. This indicates that, at its optimum location, the transfer rate of the electron from $\mathrm{I}^{-}$to the NCS is extremely fast. Thus, the QM results show that the redox potentials of $\mathrm{I}^{-}$and $\mathrm{D}^{+}$are well matched when desolvation is taken into account (vide infra). To quantify this, we evaluated the Mulliken populations at the site-SD structure for the DFT wavefunction with PBF solvation. The Mulliken spin density of the iodine atom is 0.597 ; hence, $40 \%$ of the spin has been transferred to the NCS.

Thus, our QM calculations show that $\mathrm{I}^{-}$is attracted to the NCS region of the oxidized dye, where the NCS ligand bridges two redox centers of the iodide and $\mathrm{Ru}$ for a favorable innersphere electron transfer (ISET) to reduce the dye. ${ }^{34}$

As shown in Figure 2c, the site-DD configuration does not lead to ISET to the dye. Here, the Mulliken spin of the I atom is only 0.014 , although the distances between the $\mathrm{Ru}$ atom and the I atom for site-SD and site-DD are nearly identical (5.796 vs $5.558 \AA$ ). This implies that direct charge transfer is not allowed when the iodide interacts with the $\mathrm{dcb}$, confirming the importance of NCS ligand bridging for an efficient charge transfer.

To validate the accuracy of our DFT calculations, we carried out a number of calculations using various basis sets and functions, as will be reported elsewhere (summarized in Tables S2 and S3). We find that our DFT+PBF calculations 
(employing the same functional, basis sets, and atomic radius parameters for $\mathrm{PBF}$ ) lead to $E^{0}\left(\mathrm{I}^{\bullet} / \mathrm{I}^{-}\right)=1.471 \mathrm{~V}$ (assuming that spin-orbit (SO) coupling of $\mathrm{I}^{\bullet}$ is quenched by the strongly polarizing solvents) and $1.107 \mathrm{~V}$ (including full SO corrections), which can be compared to the experimental $E^{0}\left(\mathrm{I}^{\bullet} / \mathrm{I}^{-}\right)=1.224 \pm 0.01 \mathrm{~V} \cdot{ }^{14}$ For the dye, we calculate $E^{0}\left(\mathrm{D}^{+} /\right.$ $\left.\mathrm{D}^{0}\right)=1.035 \mathrm{~V}$ (vs SHE), which is comparable to the experimental $E^{0}\left(\mathrm{D}^{+} / \mathrm{D}^{0}\right)=1.074-1.094 \mathrm{~V} .^{15,35}$ Thus we calculate the difference in the redox potentials, showing it to be $0.072 \mathrm{~V}$ (assuming no quenching of SO), compared to an experimental difference of $0.13-0.15 \mathrm{~V}$.

This unfavorable outer-sphere electron transfer (OSET) energetics has led to a great deal of consternation in the literature, with some postulating that two $\mathrm{I}^{-}$ions must be involved to have sufficient oxidizing power. For example, the TIP assumes that a $\left(\mathrm{D}^{+} \ldots \mathrm{I}^{-}\right)$complex forms first, followed by an interaction with a second $\mathrm{I}^{-}$to reduce $\mathrm{D}^{+}$with the simultaneous forming of $\mathrm{I}_{2}^{-}$. This would lead to bilinear dependencies on the $\mathrm{I}^{-}$concentration unless the strong ionpairing of $\mathrm{D}^{+}$with the first $\mathrm{I}^{-}$is established, which could yield a pseudo-first-order kinetics. Our DFT calculations led to a complexation energy of only $\sim 3.6 \mathrm{kcal} / \mathrm{mol}$, which we consider as weak ion-pairing. Indeed the transient absorption experiments lead to a first-order kinetics. ${ }^{7,12}$

Despite this apparent mismatch in the redox potentials, we find that for the solvated $\left(\mathrm{D}^{+} \cdots \mathrm{I}^{-}\right)$complex, the ISET from $\mathrm{I}^{-}$ to $\mathrm{D}^{+}$is favorable. To understand why this becomes favorable, we decompose the solution-phase electron transfer from the iodide to the oxidized dye via SIP into several steps:

$$
\begin{aligned}
& \text { Net: } \mathrm{D}^{+}(\text {soln })+\mathrm{I}^{-}(\text {soln }) \rightarrow \mathrm{D}^{0}(\text { soln })+\mathrm{I}(\text { soln }) \\
& \mathrm{D}^{+}(\text {gas })+\mathrm{I}^{-}(\text {gas }) \rightarrow \mathrm{D}^{0}(\text { gas })+\mathrm{I}^{\bullet}(\text { gas }) \\
& \mathrm{D}^{+}(\text {soln }) \rightarrow \mathrm{D}^{+}(\text {gas }) \\
& \mathrm{D}^{0}(\text { gas }) \rightarrow \mathrm{D}^{0}(\text { soln }) \\
& \left.\mathrm{I}^{-}(\text {soln }) \rightarrow \mathrm{I}^{-} \text {(gas }\right) \\
& \mathrm{I}^{\bullet}(\text { gas }) \rightarrow \mathrm{I}^{\bullet}(\text { soln })
\end{aligned}
$$

Reaction 2 is the gas-phase electron transfer from the iodide to the oxidized dye (no solvation), which is the difference in the electron affinity (EA) of the iodide and the ionization energy (IE) of the dye. Our DFT results (SO corrected) show that the reaction free energy of (2) is $\Delta G^{\mathrm{ET}(\mathrm{gas})}=-3.190 \mathrm{eV}$, i.e., large and exothermic. Thus, without considering solvation, the electron transfer would be most strongly favorable. We can visualize this by considering the energy of the p-orbital of $\mathrm{I}^{-}$to be $\sim 3.2 \mathrm{eV}$ higher than the energy of the $t_{2 g}$-like hole state of $\mathrm{D}^{+}$, as shown in Figure S6.

The reaction free energy changes required for (3) and (4) are determined by the solvation energy difference between neutral species and charged species: $\Delta G^{\circ}{ }_{s}(\mathrm{D})=G^{\circ}{ }_{s}\left(\mathrm{D}^{0}\right)-$ $G^{\circ}{ }_{s}\left(D^{+}\right)$. Similarly, the reaction free energy changes for $(5)$ and (6) can be defined as $\Delta G^{\circ}{ }_{s}(\mathrm{I})=G^{\circ}{ }_{s}\left(\mathrm{I}^{\circ}\right)-G^{\circ}{ }_{s}\left(\mathrm{I}^{-}\right)$. Since the solvation energy of a charged species is much larger than that of a neutral species, these two $\Delta G^{\circ}$ values are positive (unfavored). Our PBF calculation leads to $\Delta G^{\circ}{ }_{s}(\mathrm{D})=0.757$ $\mathrm{eV}$ and $\Delta G^{\circ}{ }_{s}(\mathrm{I})=2.504 \mathrm{eV}$. The small size of the iodine atom results in a more dramatic change in the solvation energy when the electron is lost. Thus, the overall reaction free energy for (2) becomes $\Delta G^{\mathrm{ET}(\text { gas })}+\Delta G^{\circ}{ }_{s}(\mathrm{D})+\Delta G^{\circ}{ }_{s}(\mathrm{I})=0.072 \mathrm{eV}$, which is endothermic (unfavored), as concluded from the comparison of the standard reduction potentials.

This discussion based on the overall free energy change (i.e., the standard reduction potential difference) assumes that each species forms separate complete solvation shells, which is appropriate for long-range OSET between well-separated chemical species (schematically shown in Scheme 1a). For this fully solvated system, it is no longer favorable for $\mathrm{I}^{-}$to transfer to $\mathrm{D}^{+}$, as it was for the gas phase.

Scheme 1. (a) Outer-Sphere Electron Transfer $\left(\right.$ OSET) ${ }^{a}$ and (b) Inner-Sphere Electron Transfer (ISET) ${ }^{b}$ Processes

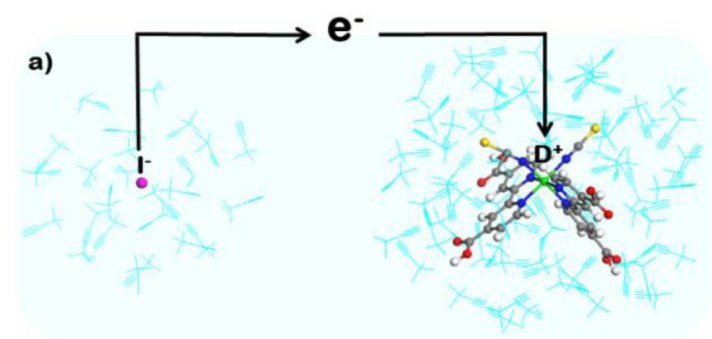

b)

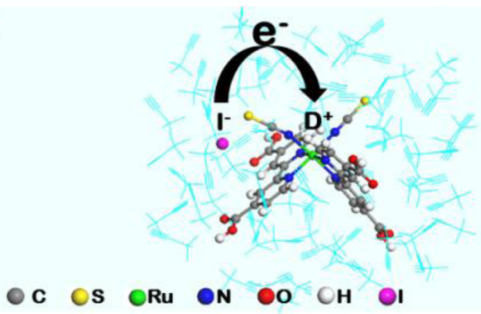

${ }^{a} \mathrm{We}$ assume that there is a complete solvation sphere around both $\mathrm{I}^{-}$ and $\mathrm{D}^{+}$, leading to a long-range OSET between well-separated chemical species. This results in a higher $E^{0}\left(\mathrm{I}^{\bullet} / \mathrm{I}^{-}\right)$than $E^{0}\left(\mathrm{D}^{+} / \mathrm{D}^{0}\right)$ by $\sim 0.072 \mathrm{~V}$ from the $\mathrm{QM}$ and $0.13-0.15 \mathrm{eV}$ from the experiment.

${ }^{b}$ At the QM equilibrium structure solvated with AN, the NCS ligand bridges I and $\mathrm{Ru}$ within the same solvation shell. As a result, $\mathrm{I}^{-}$is partially desolvated, resulting in a redox potential that matches that of the dye, enabling a fast ISET process.

However, our QM calculations show that at the equilibrium structure of the $\left(\mathrm{D}^{+} \ldots \mathrm{I}^{-}\right)$complex, the solvation of $\mathrm{I}^{-}$is reduced, making ISET favorable. Thus, after the excitation and electron transfer from the dye, migration of $\mathrm{I}^{-}$to $\mathrm{D}^{+}$leads to a favorable ISET condition. Consequently the kinetics of regeneration is limited by the diffusion of $\mathrm{I}^{-}$, leading to a microsecond order of the time scale for the dye regeneration process. ${ }^{16}$

Summarizing, the QM calculations show that under the AN solvation environment, a single iodide can transfer directly its electron to the oxidized dye, which is contrary to the claim that at least two iodides are required to reduce the dye due to the higher $E^{0}$ of iodine as compared to the $E^{0}$ of the dye. ${ }^{13-15} \mathrm{We}$ find instead that $\mathrm{I}^{-}$is partially desolvated as it is attracted into the first solvation shell at the NCS-dcb dye interface (site-SD), making it favorable for the ISET of the electron from $\mathrm{I}^{-}$to reduce the dye (as schematically shown in Scheme $1 \mathrm{~b}$ ).

A feature of the N3 dye important for this rapid ISET process is that the NCS ligand of the $\mathrm{Ru}(\mathrm{III})$ delocalizes the $\mathrm{d}_{x y}$ hole over the two NCS $\pi$-orbitals in the $x y$ plane (Figure $2 \mathrm{a}$ ). This allows the hole to overlap the p-orbital of $\mathrm{I}^{-}$at its energy minimum, where the NCS ligand bridges $\mathrm{I}^{-}$and $\mathrm{Ru}(\mathrm{III})$, leading to ISET from the electron on $\mathrm{I}^{-}$to fill the hole in $\mathrm{D}^{+}$. 
This suggests that the solid angle of the initial iodide with respect to both the NCS group and the Ru metal center should be important for an efficient electron transfer. Thus, possible strategies to improve the charge regeneration rate are as follows:

(1) Replace the NCS with a ligand that would increase delocalization onto the ligand and/or enhance the interaction with the iodide.

(2) Design bipyridinyl groups to increase the binding of both pyridine groups to the $\mathrm{TiO}_{2}$ so that the dye molecule stands perpendicular to the $\mathrm{TiO}_{2}$ surface, opening the side binding sites ( site-SD).

\section{ASSOCIATED CONTENT}

\section{(S Supporting Information}

Tables S1-S4, showing solvation energy of $\mathrm{I}^{-}$and $\mathrm{I}_{3}^{-}$, electron affinity of $\mathrm{I}_{1} \mathrm{I}_{2}$, and $\mathrm{I}_{3}$, the reduction potential of iodide-related species and dye, and reduction potential of $\left(\mathrm{Fc}^{+} / \mathrm{Fc}\right)$; Figures S1-S3, showing the initial structures of the $\left(\mathrm{D}^{+} \cdots \mathrm{I}^{-}\right)$complex, the molecular orbitals of $\mathrm{D}^{+}$, and Born-Haber cycle scheme; Figures S4-S6, showing the MO of the $\mathrm{D}^{0}$, N-bonded and Sbonded isomers of the dye molecule, and an energy diagram of $\mathrm{I}^{-}$and $\mathrm{D}^{+}$. This material is available free of charge via the Internet at http://pubs.acs.org.

\section{AUTHOR INFORMATION}

\section{Corresponding Author}

wag@wag.caltech.edu; linus16@kaist.ac.kr

\section{Notes}

The authors declare no competing financial interest.

${ }^{\S}$ World Class University Professor, EEWS, KAIST

\section{ACKNOWLEDGMENTS}

This work is supported by the World Class University program (R31-2008-000-10055-0) funded by the Ministry of Education, Science and Technology of Korea.

\section{REFERENCES}

(1) Oregan, B.; Gratzel, M. Nature 1991, 353, 737.

(2) Gratzel, M. Inorg. Chem. 2005, 44, 6841.

(3) Benko, G.; Kallioinen, J.; Korppi-Tommola, J. E. I.; Yartsev, A. P.; Sundstrom, V. J. Am. Chem. Soc. 2002, 124, 489.

(4) Haque, S. A.; Palomares, E.; Cho, B. M.; Green, A. N. M.; Hirata, N.; Klug, D. R.; Durrant, J. R. J. Am. Chem. Soc. 2005, 127, 3456.

(5) Anderson, N. A.; Lian, T. Q. In Annual Reviews in Physical Chemistry; Annual Reviews: Palo Alto, 2005; Vol. 56, p 491.

(6) Green, A. N. M.; Palomares, E.; Haque, S. A.; Kroon, J. M.; Durrant, J. R. J. Phys. Chem. B 2005, 109, 12525.

(7) Montanari, I.; Nelson, J.; Durrant, J. R. J. Phys. Chem. B 2002, 106, 12203.

(8) Haque, S. A.; Tachibana, Y.; Klug, D. R.; Durrant, J. R. J. Phys. Chem. B 1998, 102, 1745.

(9) Boschloo, G.; Hagfeldt, A. Acc. Chem. Res. 2009, 42, 1819.

(10) Yanagida, S.; Yu, Y. H.; Manseki, K. Acc. Chem. Res. 2009, 42, 1827.

(11) Nasr, C.; Hotchandani, S.; Kamat, P. V. J. Phys. Chem. B 1998, 102, 4944.

(12) Anderson, A. Y.; Barnes, P. R. F.; Durrant, J. R.; O’Regan, B. C. J. Phys. Chem. C 2011, 115, 2439.

(13) Boschloo, G.; Gibson, E. A.; Hagfeldt, A. J. Phys. Chem. Lett. 2011, 2, 3016.

(14) Wang, X. G.; Stanbury, D. M. Inorg. Chem. 2006, 45, 3415.
(15) Nazeeruddin, M. K.; Kay, A.; Rodicio, I.; Humphrybaker, R.; Muller, E.; Liska, P.; Vlachopoulos, N.; Gratzel, M. J. Am. Chem. Soc. 1993, $115,6382$.

(16) Clifford, J. N.; Palomares, E.; Nazeeruddin, M. K.; Gratzel, M.; Durrant, J. R. J. Phys. Chem. C 2007, 111, 6561.

(17) Fitzmaurice, D. J.; Frei, H. Langmuir 1991, 7, 1129.

(18) Gardner, J. M.; Abrahamsson, M.; Farnum, B. H.; Meyer, G. J. J. Am. Chem. Soc. 2009, 131, 16206.

(19) Gardner, J. M.; Giaimuccio, J. M.; Meyer, G. J. J. Am. Chem. Soc. 2008, 130, 17252.

(20) Farnum, B. H.; Jou, J. J.; Meyer, G. J. Proc. Natl. Acad. Sci. U.S.A. 2012, 109, 15628.

(21) Alebbi, M.; Bignozzi, C. A.; Heimer, T. A.; Hasselmann, G. M.; Meyer, G. J. J. Phys. Chem. B 1998, 102, 7577.

(22) Pelet, S.; Moser, J. E.; Gratzel, M. J. Phys. Chem. B 2000, 104, 1791.

(23) Nyhlen, J.; Boschloo, G.; Hagfeldt, A.; Kloo, L.; Privalov, T. Chemphyschem 2010, 11, 1858.

(24) Lobello, M. G.; Fantacci, S.; De Angelis, F. J. Phys. Chem. C 2011, 115, 18863.

(25) Schiffmann, F.; VandeVondele, J.; Hutter, J.; Urakawa, A.; Wirz, R.; Baiker, A. Proc. Natl. Acad. Sci. U.S.A. 2010, 107, 4830.

(26) Jeon, J.; Kim, H.; Goddard, W. A.; Pascal, T. A.; Lee, G. I.; Kang, J. K. J. Phys. Chem. Lett. 2012, 3, 556.

(27) Cramer, C. J.; Truhlar, D. G. Chem. Rev. 1999, 99, 2161.

(28) Maestro; Schrödinger, LLC: New York, 2009.

(29) Ritzoulis, G.; Papadopoulos, N.; Jannakoudakis, D. J. Chem. Eng. Data 1986, 31, 146.

(30) Boes, E. S.; Livotto, P. R.; Stassen, H. Chem. Phys. 2006, 331, 142.

(31) Nazeeruddin, M. K.; Humphry-Baker, R.; Liska, P.; Gratzel, M. J. Phys. Chem. B 2003, 107, 8981.

(32) Nazeeruddin, M. K.; Baranoff, E.; Gratzel, M. Sol. Energy 2011, $85,1172$.

(33) Nazeeruddin, M. K.; De Angelis, F.; Fantacci, S.; Selloni, A.; Viscardi, G.; Liska, P.; Ito, S.; Bessho, T.; Gratzel, M. J. Am. Chem. Soc. 2005, 127, 16835.

(34) Taube, H. G.; Gould, E. S. Acc. Chem. Res. 1969, 2, 321.

(35) Bond, A. M.; Deacon, G. B.; Howitt, J.; MacFarlane, D. R.; Spiccia, L.; Wolfbauer, G. J. Electrochem. Soc. 1999, 146, 648. 